\title{
COMPARISON BETWEEN CONE-BEAM COMPUTED TOMOGRAPHY AND INTRAORAL RADIOGRAPHY TO DIAGNOSE PERIODONTAL- BONE DEFECT AND TREATMENT RESPONSE
}

\author{
Seraj M Keibah*, Abdel-Fattah M Amer** and Mohammed F Khedr ${ }^{* * *}$
}

\begin{abstract}
The purpose of this study was to explore the diagnostic value of CBCT in the determination of periodontal bone loss of infrabony defects. Twenty-one non-smoking adult subjects with a total of 38, periodontal bone defects were evaluated. To be eligible to participate in the study, patients had to have: at least one infrabony defect (distance between alveolar crest and base of the defect) with interproximal probing $\geq 5 \mathrm{~mm}$, attachment loss $\geq 3 \mathrm{~mm}$ and intraoral periapical radiographs were taken to confirm the presence of suitable bony defects to be included in the study. Direct digital intraoral radiography (RVG) and CBCT were taken at the site of the bone defect. Periodontal bone defect will be measured from the cemento-enamel junction (CEJ) to the alveolar crest (AC) and from the cement-enamel junction (CEJ) to the bottom of the defect. Patients were instructed in self-performed plaque control measures, three weeks before surgery, full mouth supra-gingival and sub-gingival debridement and root planning of all quadrants were performed. Two weeks following initial cause-related therapy, periodontal reevaluation was performed to confirm those sits indicated for periodontal surgery. Periodontal flap surgery was performed for the patients. Periodontal bone defect from the cemento-enamel junction (CEJ) to the alveolar crest (AC) and from the cement-enamel junction (CEJ) to the bottom of the defect was measured as the gold standard by Williams probe (Hu-Friedy, Chicago, USA). After measurements, bone restorative steps were done if necessary and measurement were repeated after 6 months of treatment. The findings of the present investigation demonstrated a statistically significant difference between cone-beam computed tomography and intraoral radiography. In addition, a statistically significant difference between intraoral radiography and intra-surgical measurements of bone defects was detected. However, cone-beam computed tomography and intra-surgical measurements did not record the difference statistically significant difference. Furthermore, there was a high degree of correlation between intra-surgical and CBCT measurements of bone defects.
\end{abstract}

\section{INTRODUCTION}

Periodontal diseases consist of a variety of conditions affecting the periodontal tissues including: gingiva, periodontal ligament, root cementum and alveolar bone. Inflammation of periodontal tissues is a common disease which results in attachment loss as well as the destruction of alveolar bone. Diagnosis of periodontal diseases, mainly, depends on clinical signs and symptoms ${ }^{(1)}$. However, in the case of bone destruction, radiographs are valuable diagnostic tools as an adjunct to the clinical examination. The radiographs can provide key information of relevance to periodontal decision which is not being captured by clinical examination (2). Although periapical and panoramic radiographs are routinely used for the diagnosing of periodontal bone levels, they may over-or underestimate the amount of bone loss due to projection error ${ }^{(3)}$. In addition, the projection geometry of the X-ray beam may cause magnification and distortion which makes it impossible to obtain accurate diagnosis ${ }^{(4)}$.

\footnotetext{
* BDS, Faculty of Dentistry Misurata University Libya

** Professor, Faculty of Dental Medicine, Department of Periodontology, Al Azhar

University, Cairo, Egypt.

*** Professor, Faculty of Dental Medicine, Department of Periodontology, Al Azhar University, Cairo, Egypt.
} 
It is well known that intraoral radiography provides only a 2-dimensional (2D) view of 3-dimensional (3D) structures which can lead to underestimation of bone loss and errors in identifying reliable anatomical reference points ${ }^{(5,6)}$. The (2D) images possess inherent limitations which include magnification, distortion, and superimposition (7). However, these methods are limited by overlapping anatomical structures, difficulty in standardization as well as underestimating the size and occurrence of bone defects ${ }^{(8,9)}$. Cone beam computed tomography (CBCT) has been introduced as an application of CT that generates three-dimensional (3D) data at lower cost and absorbed doses than conventional CT found in the practice of medical radiology; such technique has increased rapidly in the dental field ${ }^{(10)}$. Data from the craniofacial region are often collected at higher resolution in the axial plane than those from conventional CT systems. The advantage of CBCT over intraoral radiographs owing to its capability to assess buccal and lingual surface ${ }^{(11)}$, which provided merits to CBCT scanning as a valuable imaging modality in periodontology.

Several studies have been done to evaluate the accuracy of CBCT in measuring periodontal bone loss. Overall, these studies indicate that CBCT can accurately provide 3D morphology of periodontal defect and is significantly better than conventional intra-oral radiographs ${ }^{(12,13)}$. Grimard et a $1^{(14)}$ compared direct clinical, periapical radiograph, and CBCT measurement techniques for assessing bone level changes following regenerative periodontal therapy in 35 intrabony defects. Authors found that overall; CBCT was significantly more precise and accurate than periapical radiographs and concluded that CBCT may obviate surgical reentry as a technique for assessing regenerative therapy outcomes. A study ${ }^{(15)}$ compared periapical radiographs with $\mathrm{CBCT}$ imaging in detecting and localizing alveolar bone loss. The authors concluded that CBCT offers improved visualization of the morphology of the defect. The authors had used secondary image database for CBCT comparison and did not compare CBCT measurements with clinical gold standard.

The accuracy of CBCT in the detection of horizontal periodontal bone defects has been evaluated ${ }^{(16)}$. They measured 72 defects in maxillary molar region in patients with periodontitis using $\mathrm{CBCT}$ and direct clinical measurement performed during surgical intervention. The authors found that CBCT accurately reproduced the clinical measurement of horizontal periodontal bone defects. However, this study did not evaluate the accuracy of CBCT in vertical defects measurement. Thus, data from these studies have validated the accuracy of CBCT measurements. However, these studies had several inadequacies such as limited sample size and use of different techniques to do direct clinical measurements. And, therefore, the clinical data on the accuracy of $\mathrm{CBCT}$ as periodontal diagnostic aid is still inadequate. Studies of the extent of vertical alveolar bone defects from radiographs and from exploratory surgery have also indicated a good agreement between the radiographic and the clinical findings ${ }^{(17,18)}$. It was reported that ${ }^{(19)}$ conventional radiographic images provided a better resolution of the bone levels than what can be achieved from computer screen images. For the detection of smallest osseous defects, CBCT can display the image in all its three dimensions by removing the disturbing anatomical structures and making it possible to evaluate each root and surrounding bone. In view of this, it would be of value to compare between the validity of these advanced radiographic technologies to proper assess the of hard tissue changes induced by periodontal destruction.

\section{SUBJECTS AND METHODS}

\section{Patients Selection}

The participants were selected from the out patients clinical of the Department of Oral Medicine, Periodontology, Oral Diagnosis and Oral Radiology. 
The age of the patients was ranged from 23 to 46 years. Patients had to have: at least one intrabony defect (distance between alveolar crest and base of the defect) with interproximal probing $\geq 5 \mathrm{~mm}$, attachment loss $\geq 3 \mathrm{~mm}$ and intraoral periapical radiographs were taken to confirm the presence of suitable bony defects to be included in the study. Patients should be available whenever necessary for re-evaluation. 38 sites of osseous defects were assessed by intraoral radiography and cone-beam computed tomography (CBCT), to evaluate the architecture of alveolar bone destruction involves periodontium. Patient treated with various forms of periodontal surgery were evaluated for the response to treatment after 6 months post-surgery.

\section{Consent form:}

The nature of the study was explained to the patients and they were singe a consent form before study.

\section{Inclusion criteria:}

The patients who had all the inclusion criteria were selected. 1) Interproximal osseous defects (horizontal or 3 walls vertical), 2) Indication for periodontal surgery at the site of the defect, 3) No contraindication of periodontal surgery, 4) No contraindication to radiography.

\section{Exclusion criteria:}

1. Patient under 18 years of age, 2. History of excessive radiation exposure. 3. Uncontrolled systemic disease.4. Pregnancy. 5. Presence of large full coverage restorations and restoration located close to the cementoenamel junction. 6.1Smokers.

\section{Radiographic Evaluation}

After enrolling the patients in the study, direct digital intraoral radiography (RVG Kodak sensor size 1 with specific software, CS 3D imaging version 3.2.12) and CBCT (Kodak 9500 CBCT scanner) were taken at the site of the bone defect. In direct digital intraoral radiography, intraoral digital images were obtained using the paralleling technique in a standardized exposure set-up. One technician took all the radiographies to reduce interfering factors. Images were seen and measured by software and saved as JPEG format.

For CBCT radiographs, images were taken with Kodak 9500 CBCT scanner voxel size of half skull program 200 micron and full skull program 300 micron. Images were examined with specific software (CS 3D imaging version 3.2.12) in a PC workstation running under Microsoft Windows XP Professional SP-2 (Microsoft Corp, Redmond, WA). CBCT images were displayed on a computer screen (LCD monitor) and visual analysis of all images were read by an experienced radiologist performed. Software was used in coronal and sagittal plans to reconstruct observation images.

\section{Radiographic Measurements}

Images were saved as JPEG files to measure by observers, and then by special software ruler. These specifications were considered for CBCT. Regarding these two radiographic modalities, two observers evaluated images and randomly measured periodontal bone levels and classified the defects. Measurement tools on both programs were used for assessing bone levels. Vertical dimension of the periodontal bone defects was measured under standard conditions and constant environment (same monitor, without changes in contrast and resolution, same lightness of room, and equal distance from the monitor). Periodontal bone defect will be measured from the cemento-enamel junction (CEJ) to the alveolar crest (AC) and from the cement-enamel junction (CEJ) to the bottom of the defect (Figure 1).

\section{Presurgical mouth preparation:}

All patients were instructed in self-performed plaque control measures using soft tooth brush and interdental cleaning devices. Three weeks before surgery, full mouth supra-gingival scaling and sub-gingival debridement and root planning 
of all quadrants were performed. Scaling and root planning were performed in quadrants using a combination of hand and ultrasonic instruments until the surface was clean, hard, and smooth as determined by explorer. Patients were received detailed mechanical plaque control instructions. Two weeks following initial cause-related therapy, periodontal reevaluation was performed to confirm those sits indicated for periodontal surgery.

\section{Surgical Treatment}

Open flap surgery was performed for the prepared patients according to the indication. For selection of appropriate technique, observation and access to the alveolar crest and depth of the defect

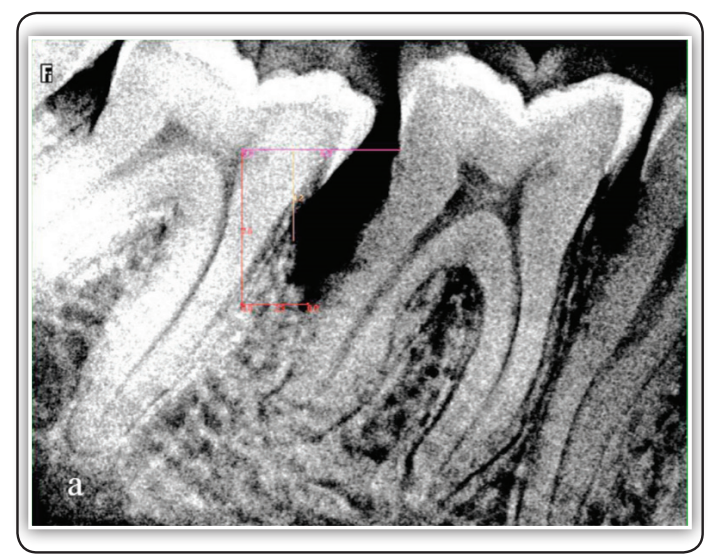

FIG (1) a, Intra oral radiograph measurement from the CEJ to $\mathrm{BD} \&$ from CEJ to AC

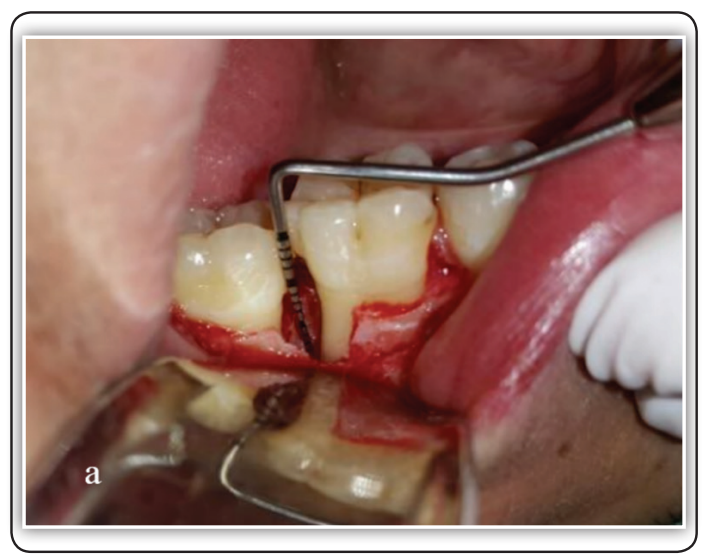

FIG (2) a, Measurement of the vertical bone defect during surgery from CEJ- BD were considered. Surgery was performed at planed time under local anesthesia. After incision, flap was elevated and granulation tissue was removed to increase the access; scaling and root planning was done if necessary. Periodontal bone defect from the cemento-enamel junction (CEJ) to the alveolar crest (AC) and from the cement-enamel junction (CEJ) to the bottom of the defect was measured as the gold standard by Williams probe (Hu-Friedy, Chicago, USA) (Figure 2). The acquired measurement data and periodontal defect classifications were compared with the gold standard. After measurements, bone restorative steps were done if necessary and measurement were repeated after 6 months of treatment (Figure 3).

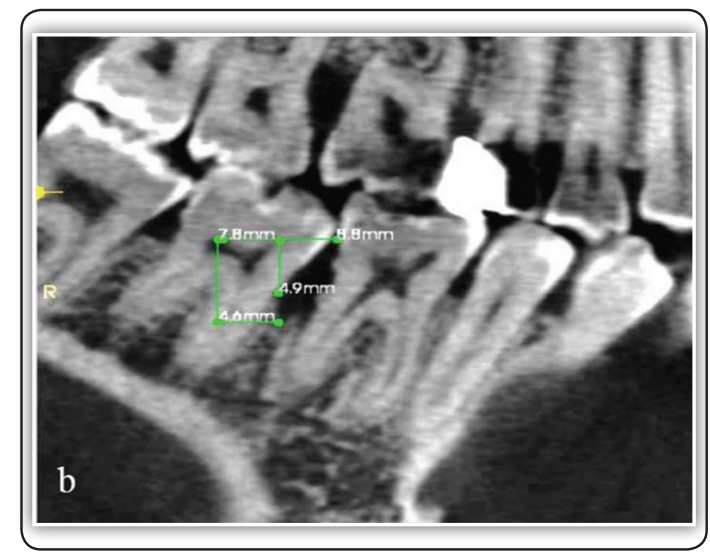

FIG (1)b, CBCT Measurement from the CEJ-to BD \& from $\mathrm{CEJ}$ to $\mathrm{AC}$

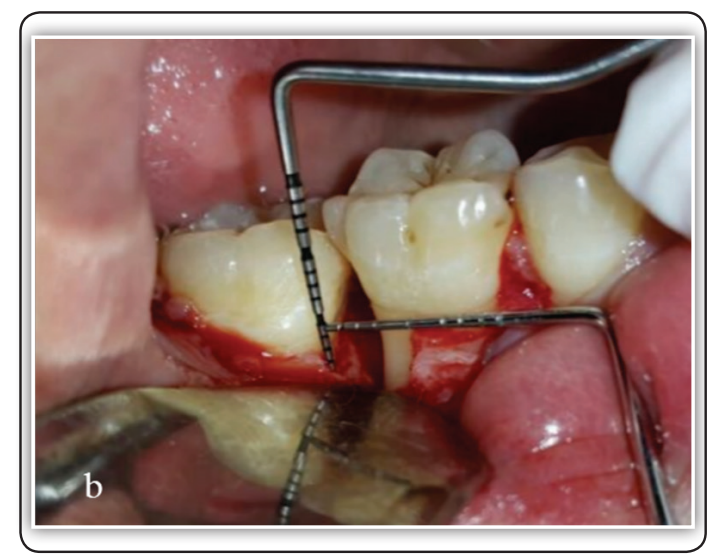

FIG (2)b, measurement of bone defect during surgery from CEJ - AC 


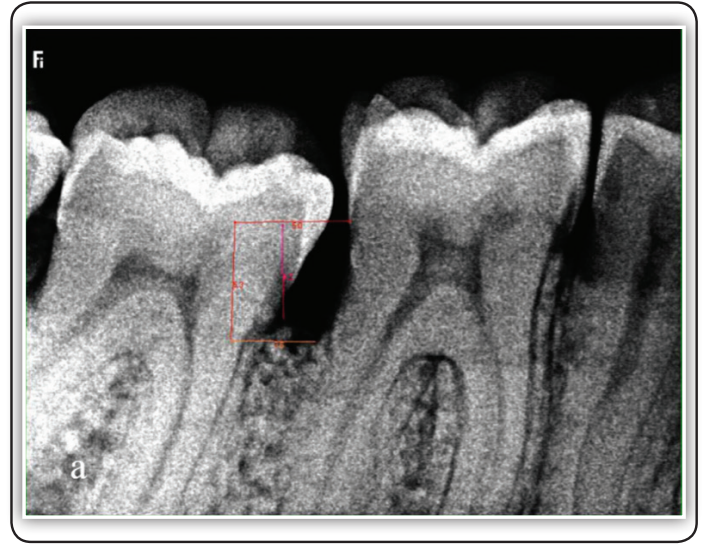

FIG (3) a, Post treatment Intra oral radiograph measurement from the CEJ-to BD \& from CEJ to AC

\section{Statistical analysis}

Paired t-test was used for the comparing between Periapical radiograph and CBCT as well as for comparing between pre and post treatment. Correlation between periapical radiograph, CBCT and Intra-Surgical Was done by Pearson coefficient. Statistically significant was set at $\mathrm{p} \leq 0.05$.

\section{RESULTS}

Paired t-test was used for the comparing between periapical radiograph and CBCT as well as for comparing between pre and post treatment. Correlation between periapical radiograph, CBCT and Intra-Surgical Was done by Pearson coefficient. Statistically significant was set at $\mathrm{p} \leq 0.05$.

1. Comparison between periapical radiograph, CBCT and Intra-Surgical in cemento enamel junction to the body of defect (CEJ-BD)

As regard to CEJ-BD measure, there was a statically significant difference between Periapical radiograph and CBCT $(\mathrm{p}<0.05)$. Also, statically significant difference was found between Periapical radiograph and Intra-Surgical $(\mathrm{p} \leq 0.05)$, while statically non-significant value for comparing between CBCT and Intra-Surgical was rated [Table 1].

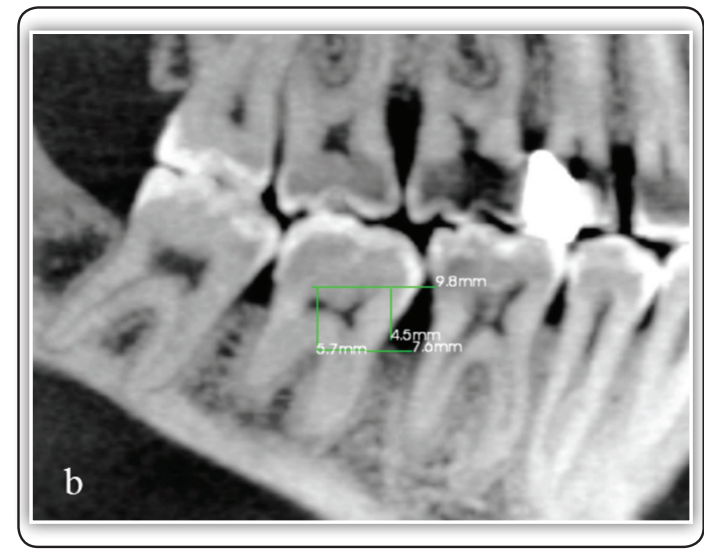

FIG (3) b, post treatment CBCT measurement from the CEJ to $\mathrm{BD} \&$ from $\mathrm{CEJ}$ to $\mathrm{AC}$

TABLE (1) Comparison between periapical radiograph, CBCT presurgical and Intra-Surgical in cemento enamel junction to the body of defect (CEJBD) $(\mathrm{n}=38)$

\begin{tabular}{|c|c|c|c|}
\hline & $\begin{array}{c}\text { Periapical } \\
\text { radiograph }\end{array}$ & CBCT & \multicolumn{1}{|c|}{$\begin{array}{c}\text { Intra } \\
\text { Surgical }\end{array}$} \\
\hline CEJ-BD & \multicolumn{3}{|l|}{} \\
\hline Min. - Max. & $3.5-9.0$ & $4.0-10.50$ & $4.0-11.0$ \\
\hline Mean \pm SD. & $5.20 \pm 1.52$ & $6.09 \pm 1.61$ & $6.21 \pm 1.69$ \\
\hline $\mathbf{p}$ & $\mathrm{p}_{1}<0.001^{*}, \mathrm{p}_{2}<0.001^{*}, \mathrm{p}_{3}=0.084$ \\
\hline
\end{tabular}

$p: p$ value for Paired t-test

$p_{1}: p$ value for comparing between periapical radiograph and $C B C T$

$p_{2}: p$ value for comparing between periapical radiograph and Intra-Surgical

$p_{3}: p$ value for comparing between CBCT and Intra-Surgical

*: Statistically significant at $p \leq 0.05$

2. The Comparison between periapical radiograph, CBCT and Intra-Surgical in cemento enamel junction to the alveolar crest (CEJ- AC)

As regard to CEJ-AC measurements, there was a statically significant $(\mathrm{p} \leq 0.05)$ difference between Periapical radiograph and CBCT. Results showed 
statically significant difference $(\mathrm{p} \leq 0.05)$ between Periapical radiograph and Intra-Surgical, while statically non-significant difference between CBCT and Intra-Surgical [Table 2].

TABLE (2) The Comparison between periapical radiograph, CBCT pre-surgical and Intra-Surgical in cemento enamel junction to the alveolar crest $(\mathrm{CEJ}-\mathrm{AC})(\mathrm{n}=38)$

\begin{tabular}{|c|c|c|c|}
\hline & $\begin{array}{c}\text { Periapical } \\
\text { radiograph }\end{array}$ & CBCT & $\begin{array}{c}\text { Intra- } \\
\text { Surgical }\end{array}$ \\
\hline CEJ_AC & \multicolumn{2}{|l|}{} \\
\hline Min.-Max. & $1.50-5.50$ & $2.20-5.70$ & $2.0-6.0$ \\
\hline Mean \pm SD. & $3.31 \pm 1.06$ & $3.84 \pm 0.96$ & $4.0 \pm 1.0$ \\
\hline P & \multicolumn{2}{|c|}{$\mathrm{p}_{1}<0.001^{*}, \mathrm{p}_{2}<0.001^{*}, \mathrm{p}_{3}=0.059$} \\
\hline
\end{tabular}

$p: p$ value for Paired $t$-test

$p_{I}: p$ value for comparing between periapical radiograph and $C B C T$

$p_{2}: p$ value for comparing between periapical radiograph and Intra-Surgical

$p_{3}: p$ value for comparing between $C B C T$ and Intra-Surgical

*: Statistically significant at $p \leq 0.05$

\section{Correlation between periapical radiograph,} CBCT pre-surgical and Intra-Surgical

Results are shown in table 3 .

TABLE (3) Correlation between periapical radiograph, CBCT and Intra-Surgical

\begin{tabular}{|l|c|c|c|c|}
\hline & \multicolumn{2}{|c|}{ CEJ-BD } & \multicolumn{2}{c|}{ CEJ-AC } \\
\hline & $\mathrm{r}$ & $\mathrm{p}$ & $\mathrm{r}$ & $\mathrm{p}$ \\
\hline $\begin{array}{l}\text { Periapical radiograph vs } \\
\text { CBCT }\end{array}$ & $0.975^{*}$ & $<0.001$ & $0.947^{*}$ & $<0.001$ \\
\hline $\begin{array}{l}\text { Periapical radiograph vs } \\
\text { Intra Surgical }\end{array}$ & $0.970^{*}$ & $<0.001$ & $0.894^{*}$ & $<0.001$ \\
\hline CBCT vs Intra-Surgical & $0.982^{*}$ & $<0.001$ & $0.931^{*}$ & $<0.001$ \\
\hline
\end{tabular}

r: Pearson coefficient

*: Statistically significant at $p \leq 0.05$
4. The Comparison between Periapical radiograph and CBCT in cemento enamel junction to body of defect before and after treatment (CEJ -BD)

Regarding the results of CEJ-BD measure, there was a statically significant $(\mathrm{p} \leq 0.05)$ difference between Periapical radiograph and CBCT before and after treatment [Table 4].

TABLE (4) The Comparison between periapical radiograph and CBCT in cemento enamel junction to body of defect before and after Treatment (CEJBD) $(\mathrm{n}=38)$

\begin{tabular}{|c|c|c|c|}
\hline CEJ-BD & $\begin{array}{l}\text { Periapical } \\
\text { radiograph }\end{array}$ & CBCT & $\mathbf{p}_{1}$ \\
\hline \multicolumn{4}{|l|}{ Pre } \\
\hline Min. - Max. & $3.50-9.0$ & $4.0-10.50$ & \multirow{2}{*}{$<0.001^{*}$} \\
\hline Mean \pm SD & $5.20 \pm 1.52$ & $6.09 \pm 1.61$ & \\
\hline \multicolumn{4}{|l|}{ Post } \\
\hline Min. - Max. & $1.50-6.0$ & $2.20-8.50$ & \multirow{2}{*}{$0.001^{*}$} \\
\hline Mean \pm SD & $3.97 \pm 1.19$ & $4.60 \pm 1.43$ & \\
\hline $\mathbf{p}_{2}$ & $<0.001^{*}$ & $<0.001^{*}$ & \\
\hline
\end{tabular}

$p_{1}: p$ value for paired t-test for comparing between periapical radiograph and $C B C T$

$p_{2}: p$ value for paired t-test for comparing between pre and post

*: Statistically significant at $p \leq 0.05$

\section{Comparison between Periapical radiograph} and $\mathrm{CBCT}$ in cemento enamel junction to the alveolar crest (CEJ_AC) before and after treatment

CEJ-AC measurement, showed a statically significant $(\mathrm{p} \leq 0.05)$ difference between Periapical radiograph and $\mathrm{CBCT}$ before and after treatment [Table 5]. 
TABLE (5) The Comparison between periapical radiograph and CBCT in cemento enamel junction to the alveolar crest (CEJ-AC) before and after treatment $(\mathrm{n}=38)$

\begin{tabular}{|c|c|c|c|}
\hline CEJ-AC & $\begin{array}{l}\text { Periapical } \\
\text { radiograph }\end{array}$ & CBCT & $p_{1}$ \\
\hline \multicolumn{4}{|l|}{ Pre } \\
\hline Min. - Max. & $1.50-5.50$ & $2.20-5.70$ & \multirow{2}{*}{$<0.001^{*}$} \\
\hline Mean \pm SD & $3.31 \pm 1.06$ & $3.84 \pm 0.96$ & \\
\hline \multicolumn{4}{|l|}{ Post } \\
\hline Min. - Max. & $1.20-4.50$ & $1.40-5.0$ & \multirow{2}{*}{$<0.001^{*}$} \\
\hline Mean \pm SD & $2.80 \pm 1.12$ & $3.43 \pm 1.11$ & \\
\hline $\mathbf{p}_{2}$ & $<0.001^{*}$ & $0.023^{*}$ & \\
\hline
\end{tabular}

$p_{1}: p$ value for Paired t-test for comparing between periapical radiograph and $C B C T$

$p_{2}: p$ value for Paired t-test for comparing between pre and post

*: Statistically significant at $p \leq 0.05$

\section{DISCUSSION}

Extra oral and intra oral Radiography plays an important role in periodontal diagnosis as they reveal the amount and type of damage caused to the alveolar bone. A number of intraoral and extra oral imaging modalities are available to assist in the management of the periodontal diseases. Commonly used modalities include bitewing, periapical and panoramic radiography. However, radiographs give a 2-D representation of 3-D structures, and their limitations have been well described. There is ample research demonstrating that lingually located defects cannot be detected and that destruction of the buccal plate can be undiagnosed or undistinguished from lingual defects ${ }^{(19,20)}$. In addition, the exact form of many periodontal defects including hemiseptum, intrabony defects, and furcation involvements cannot be determined from radiographs. Surgical entry is the best way in detecting the number of walls present or absent. These limitations reduce the sensitivity of intraoral periapical (IOPA) and generally result in underestimating actual bone loss even when high quality images are produced.

To overcome the inherent difficulties of IOPA, 3-D image analysis cone beam computed tomography has been introduced and is widely used for 3-D maxillofacial imaging. It gives the morphologic description of bone defects, measures intrabony defects in all the three planes (Sagittal, Axial, Coronal), pre-surgical implant planning for anatomic land marks, measures bone volume quality of hard tissue and in periodontal aspect to visualize interproximal defects, buccal and lingual defects, furcation defects, diagnosing dehiscence and fenestration defects, diagnostic and treatmentoutcome evaluations of periodontitis, to evaluate postsurgical results of regenerative periodontal therapy, it can also be used as a new volumetric imaging method for measuring alveolar bone density, especially to assess healing after grafting ${ }^{(21)}$. The present study compared between cone-beam computed tomography and intraoral radiography in diagnosis of periodontal bone defect.

It was hypothesized that $\mathrm{CBCT}$ measurements will provide a better assessment of the depth of intra-bony defects. This study used direct intrasurgical measurement as the gold standard. The specific goal of this study was to compare intrasurgical measurement and measurements taken on a small field of view CBCT in order to establish how accurate CBCT is as a tool for evaluation of depth of intra-bony defects. In the present study a total of 38 defects were evaluated. The results showed a statistically significant difference between cone-beam computed tomography and intraoral radiography. There was, also, a statistically significant difference between intraoral radiography and intra-surgical measurements of bone defects. While cone-beam computed tomography and intra-surgical measurements the difference was statistically non-significant. Furthermore, there was a high degree of correlation between intra-surgical 
and CBCT measurements of bone defects. The results indicated that $\mathrm{CBCT}$ is highly accurate for diagnosing periodontal bone defect. These results are consistent with the results of previous studies ${ }^{(14-16)}$. These studies have evaluated and compared CBCT measurements of periodontal defects with actual surgical measurements.

Studies ${ }^{(12,13)}$ were conducted to compare between intra-bony defects in skulls using periapical or panoramic, and CBCT images; it was evident that CBCT showed closest to the true depth of the defect. Thus, it was found that CBCTs overestimated the depth of the defect by an average on $0.41 \mathrm{~mm}$. However, the results of this study were not consistent with those done on skull models. The present study in accordance with a study ${ }^{(13)}$ stated that CBCT would allow accurate assessment of bone levels and a better description of infrabony defects than intraoral CCD images. CBCT has perfect diagnostic accuracy in diagnosis of periodontal interradicular bony defects. Attempting to identify the most accurate method of evaluating hard tissue changes after periodontal therapy is an important task. To date, surgical reentry procedures appear to be the gold standard as it provides definitive information on morphology and dimensions of the infrabony defect before and after grafting. They are safe and have the advantage of being able to retreat the grafted area if complete defect resolution has not occurred. Another disadvantage of using reentry procedures in clinical research is that the recruitment of study patients and approval from Institutional Review Boards may be more difficult than less invasive means of outcome assessment. The image obtained with $\mathrm{CBCT}$, combined with various techniques such as assessment of clinical probing depths, attachment levels, and alveolar bone levels, may increase our ability to determine the treatment outcome following periodontal treatment, without the use of a reentry procedure $^{(14)}$.

In the present study, it was able to confirm the proposed hypothesis that CBCT would allow accurate assessment of bone levels and a better description of intra-bony defects than intraoral CCD images. The results show a more precise measurement deviation from the gold standard using CBCT cross-sectional slices. This finding indicated that the current $\mathrm{CBCT}$ system may become more effective in the diagnosis of periodontal diseases. Given the high accuracy of CBCT in detection of periodontal bone defects as found in the present study along with its various advantages such as low radiation, rapidity of scan time, and relatively low cost of CBCT, its use is highly desirable in periodontal practice especially for advanced periodontal disease to more accurately diagnose periodontal disease and its aspects such as amount of bone loss, involvement of furcation, type of defects and their dimension, determine accurately the prognosis of each tooth by allowing 3D analysis of bone around them, and plan for the type of periodontal intervention procedure especially related to regeneration. Also, CBCT may alleviate the need for surgical re-entry to assess bone formation. All these aspects will eventually contribute to significantly improve the quality of periodontal care and thus to improved outcome. Therefore, it may not be farfetched to speculate that in near future CBCT may replace the traditional panoramic radiographs and full mouth IOPA for periodontal diagnosis and treatment planning.

\section{REFERENCES}

1. Highfield J. Diagnosis and classification of periodontal disease. Aust Dent J 2009; 54: 11-26.

2. Bukel A, Kivanc K. Use of cone beam computed tomography in periodontology. World J Radiol 2014; 6:139-47.

3. Vandenberghe B, Jacobs R, Yang J. Detection of periodontal bone loss using digital intraoral and cone beam computed tomography image: an in vitro assessment of bony and/or infrabony defect. Bri Institute Radi 2008; 37:252-60.

4. Faria V, Evangelista K, Rodrigues C, Estrela C, Desousa $\mathrm{T}$. detection of periodontal bone loss using cone beam CT and intraoral radiography. Dentomaxillofac Radiol 2012; 41: 64-9. 
5. Ashok B, Punit S, Michael M, Michelle A, Josef S, Arnold $\mathrm{G}$, James R. Comparison of cone beam computed tomography and periapical radiography in predicting treatment decision for periapical lesions: A Clinical Study. Intern J Dent 2012; 10:8.

6. Peter E, Hausmann E. Accuracy of radiographic assessment of interproximal bone loss in intrabony defect using liner measurement. Ero J Oral Sci 2000; 108:70-3.

7. Neha K, Rahul K. Cone beam computed tomography: A thired eye dental practitioners. Inter J Stoma Res 2015; $4: 1-7$

8. Nilsun B, Mehmet E, Sebnem K, Kaan O. Comparsion of intraoral radiography and cone beam computed tomography for the detection of periodontal defect: an in vitro study. BMC Oral Health 2015; 15:64.

9. Corbet E, Dkl H, Lai S. Radiographis in periodontal disease diagnosis and management. Aust Dent J 2009; 54:27-43.

10. Donald A, Tyndall, Sonali R. Cone-Beam CT Diagnostic Application: Caries, Periodontal Bone Assessment, and Endodontic Applications. Dent Clin N Am 2008; 825-84.

11. Otavio S, Bruno F, Felipe F, Marcelo G. Detection of simulated incipient furcation involvement by CBCT: an in vitro study using pig mandibles. Brazilian Oral Res 2008; $26: 4$.

12. Misch KA, Yi ES, Sarment DP. Accuracy of cone beam computed tomography for periodontal defect measurements. J Periodontol 2006;77: 261-6.

13. Vandenberghe B, Jacobs R, Yang J. Detection of periodontal bone loss using digital intraoral and cone beam computed tomography images: An in vitro assessment of bony and/or infrabony defects. Dentomaxillofac Radiol 2008; $37: 252-60$
14. Grimard BA, Hoidal MJ, Mills MP, Mellonig JT, Nummikoski PV, Mealey BL. Comparison of clinical, periapical radiograph, and cone-beam volume tomography measurement techniques for assessing bone level changes following regenerative periodontal therapy. J Periodontal 2009; 80:48-55

15. De Faria Vasconcelos K, Evangelista KM, Rodrigues CD, Estrela C, de Sousa TO, Silva MA. Detection of periodontal bone loss using cone beam CT and intraoral radiography. Septomaxillae Radial 2012; 41:64-9.

16. Reijo CV, Lucena JG, Kurita LM, Pereira SL. Evaluation of cone beam computed tomography in the detection of horizontal periodontal bone defects: An in vivo study. Int J Periodontics Restorative Dent 2012; 32:162-8.

17. Bolin A, S. Lavstedt, L. Frithiof, and C. O. Henrikson, "Proximal alveolar bone loss in a longitudinal radiographic the progress in individuals with at least 20 remaining teeth," Acta Odontologica Scandinavica 1986;44:263-69.

18. Persson R. E., L. G. Hollender, and G. R. Persson, “Assessment of alveolar bone levels from intraoral radiographs in subject between ages 15 and 94 years seeking dental care," J Clinl Perio 1998;25: 647-54

19. Theilade J. An evaluation of the reliability of radiographs in the measurement of bone loss in periodontal disease. $\mathrm{J}$ Periodontol. 1960; 31:143-53

20. Ramadan AB, Mitchell DF. A roentgenographic study of experimental bone destruction. Oral Surg Oral Med Oral Pathol. 1962; 15:934-43

21. Ramachandran GN, Lakshminarayanan AV. Three-dimensional reconstruction from radiographs and electron micrographs: Application convolutions instead of Fourier Transforms. Proc Natl Acad Sci. 1971; 68:2236-40. 
